Research Article

Human and Medical Genetics

\title{
Integrated analysis of gene expression and DNA methylation datasets identified key genes and a 6-gene prognostic signature for primary lung adenocarcinoma
}

\author{
Jing Meng ${ }^{1 \#}$, Lei $\mathrm{Cao}^{2 \#}$, Huifang Song ${ }^{3 \#}$, Lichun $\mathrm{Chen}^{1}$ and Zhiguo $\mathrm{Qu}^{1}$ (i) \\ Inner Mongolia People's Hospital, Department of Stomatology, Hohhot, China. \\ ${ }^{2}$ Inner Mongolia People's Hospital, Department of Clinical Medical Research Center, Hohhot, China. \\ ${ }_{3}^{3}$ Inner Mongolia People's Hospital, Department of Respiratory and Critical Care Medicine, Hohhot, China.
}

\begin{abstract}
Lung adenocarcinoma (LUAD) is the main subtype of non-small cell lung cancer with a poor survival prognosis. In our study, gene expression, DNA methylation, and clinicopathological data of primary LUAD were utilized to identify potential prognostic markers for LUAD, which were recruited from The Cancer Genome Atlas (TCGA) database. Univariate regression analysis showed that there were 21 methylation-associated DEGs related to overall survival (OS), including 9 down- and 12 up-regulated genes. The 12 up-regulated genes with hypomethylation may be risky genes, whereas the other 9 down-regulated genes with hypermethylation might be protective genes. By using the Step-wise multivariate Cox analysis, a methylation-associated 6-gene (consisting of CCL20, F2, GNPNAT1, NT5E, B3GALT2, and VSIG2) prognostic signature was constructed and the risk score based on this gene signature classified patients into high- or low-risk groups. Patients of the high-risk group had shorter OS than those of the low-risk group in both the training and validation cohort. Multivariate Cox analysis and the stratified analysis revealed that the risk score was an independent prognostic factor for LUAD patients. The methylation-associated gene signature may serve as a prognostic factor for LUAD patients and the represent hypermethylated or hypomethylated genes might be potential targets for LUAD therapy.
\end{abstract}

Keywords: Lung adenocarcinoma, prognosis, signature, overall survival, risk score.

Received: December 15, 2020; Accepted: August 20, 2021.

\section{Introduction}

Lung adenocarcinoma (LUAD) is one major subtype of non-small-cell lung cancer (NSCLC) with high mortality (Siegel et al., 2015; Gharibvand et al., 2017). Because of being asymptomatic in the early phase and the delay of diagnosis, the 5 -year survival rate is $10.3 \%$ in the patients with LUAD ( $\mathrm{Li}$ et al., 2016). In view of this, it is urgent to develop a reliable biomarker to predict the prognosis of LUAD.

DNA methylation is an epigenetic process involving the addition of a methyl group to DNA. The methylation of DNA has been demonstrated to play an important role in a variety of cellular processes and disordered methylation patterns have been shown to associate with several human diseases, including cancer. Because of the stability, reversibility, and easy detectability, DNA methylation has obtained clinical attention as a novel biomarker for diagnosis and prognosis of cancer (Hao et al., 2017; Xu et al., 2017), including lung cancer (Brock et al., 2008). DNA methylation in cancer always occurs in the $\mathrm{CpG}$ islands that were presented in the

Send correspondence to Zhiguo Qu. Inner Mongolia People's Hospital, Department of Stomatology, No. 20, Zhaowuda Road, Hohhot 010010, Inner Mongolia, China. E-mail: nmgqzg@126.com.

\#These authors contributed equally to this work. promoters of a gene (Yamashita et al., 2018). As a result, these methylated $\mathrm{CpG}$ sites could affect the activation of the promoter and control the expression of the corresponding gene. Typically, high methylation of a gene always inhibits its expression, but in some cases, high methylation has been observed to promote gene expression. Studies have shown that alterations in the expression of methylation-related genes are common in the development and progression of tumors (Sheikhnejad et al., 2013; Swm et al., 2021). Furthermore, methylation-associated genes could be used to predict the prognosis of several cancer types. Although the methylationassociated genes have been demonstrated to be altered in LUAD tissue (Selamat et al., 2012; Bjaans et al., 2016; Pu et al., 2016), the prognosis value of these genes has not been well studied in LUAD.

In the current study, an integrated analysis of gene expression and DNA methylation datasets from the TCGA database was performed to identify methylation-associated prognostic genes for LUAD. A methylation-associated 6-gene signature was constructed and validated, which might contribute to improving the prognosis of LUAD patients, and might be potential targets for LUAD therapy.

\section{Material and Methods}

All data analyses were conducted relying on $\mathrm{R}$ (http:// www.r-project.org/, version 3.5.1). The analysis process is exhibited in Figure 1 as a flow chart. 


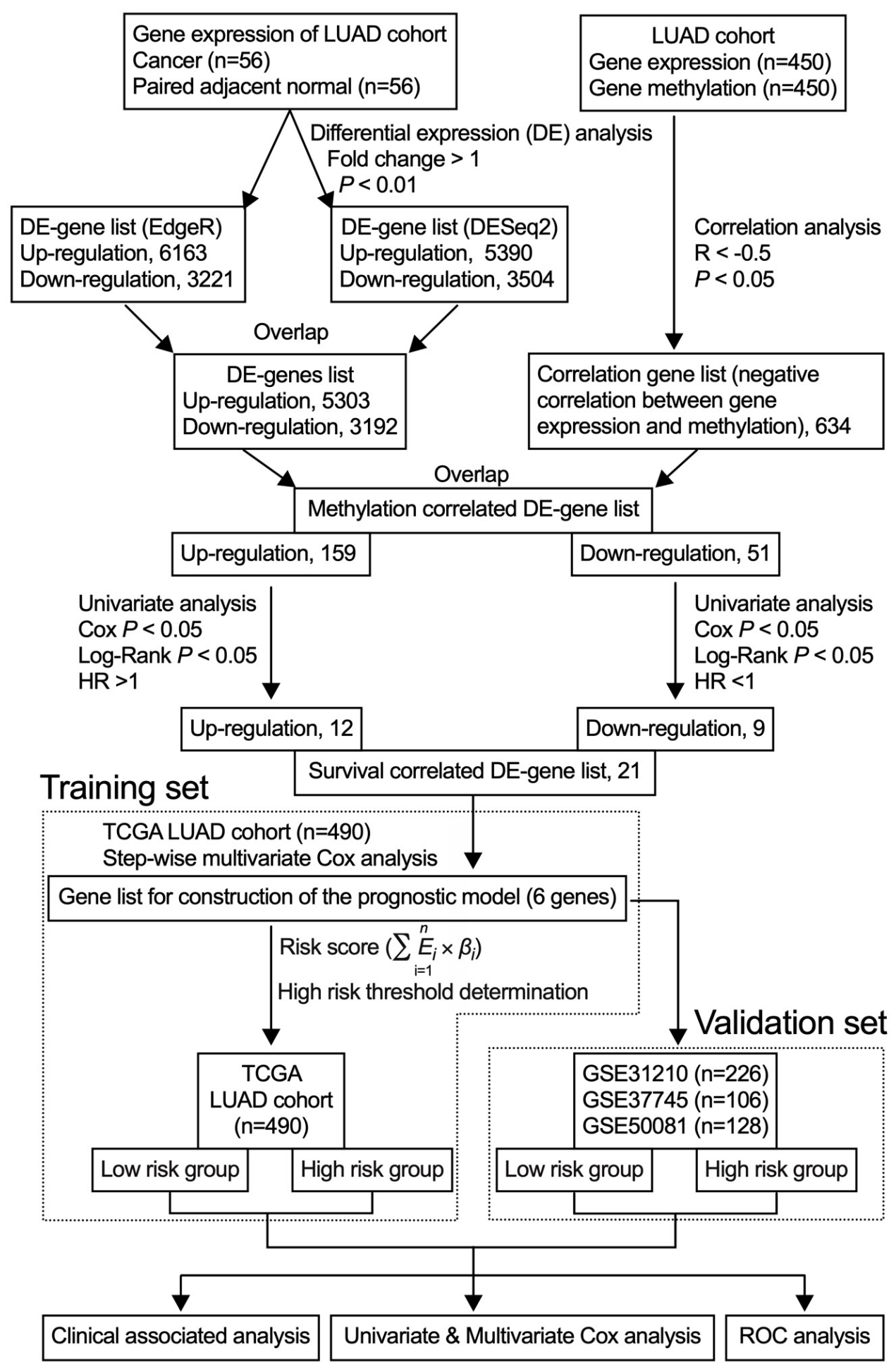

Figure 1 - Flow diagram of our study. In parentheses are the numbers of patients in each cohort. All data analyses were conducted in R.

\section{Data source}

Level 3 gene expression (RNA-seq) data, DNA methylation data, and the corresponding clinical information of the primary LUAD patients were retrieved from the TCGA database (https://portal.gdc.cancer.gov) in September, 2018. The detailed clinical data are shown in Table 2. Methylation data were based on the Illumina Infinium Human Methylation $450 \mathrm{k}$ BeadChip. A total of 490 primary LUAD samples were available and selected for further analysis. Among these 490 patients, 56 patients had paired adjacent-normal samples and 450 patients had the DNA methylation data. The expression profiles of LUAD patients under the accession number GSE31210 (Okayama et al., 2012), GSE50081 (Der et al., 2014), and GSE37745 (Botling et al., 2013) from the Gene Expression Omnibus (GEO) database (http://www.ncbi. nlm.nih.gov/gds/?term=) were downloaded as independent validation datasets. There were 226 patients in GSE31210, 106 patients in GSE37745, and 128 patients in GSE50081. 


\section{Screening for differentially expressed genes (DEGs)}

EdgeR (Robinson et al., 2009) and DESeq2 (Anders and Huber, 2010) Bioconductor packages of $\mathrm{R}$ were utilized to evaluate the DEGs between tumor and 56 paired adjacentnormal samples. Then, Benjamini and Hochberg approach was utilized to adjust the $\mathrm{P}$ values to false discovery rates (FDR) (Benjamini et al., 2001). The DEGs were identified based on $\mid$ logarithmic fold change $\mid>1$, and FDR $<0.01$, respectively. Volcano plots and scatter plots were generated using the ggplot2 package (http://ggplot2.org/), Venn Diagrams were plotted using the VennDiagram package (Chen and Boutros, 2011), while heatmaps were created using the pheatmap package (https://cran.r-project.org/web/packages/pheatmap/index.html).

\section{Correlation analysis between RNA-seq and DNA methylation}

A correlation of gene expression and DNA methylation was estimated using Pearson's correlation methods. The correlation coefficient $(\mathrm{R})<-0.5$ and $\mathrm{P}<0.05$ were used as the threshold for obtaining a list of genes in which gene expression was inversely correlated with methylation.

\section{Identification of prognosis-related signatures and calculating risk score}

The intersections of up-regulated/down-regulated genes and gene list of negative correlation between gene expression and DNA methylation levels were selected as candidates for survival analysis. Then, a univariate Cox model was applied to determine the relationship between the expression level of each candidate DEGs and OS in LUAD patients to investigate which DEGs could be served as prognostic predictors for LUAD. After that, only the DEGs with a $\mathrm{P}$ value $<0.05$ and hazard ratio $(\mathrm{HR})>1$ for up-regulated genes or $\mathrm{HR}<1$ for down-regulated genes were screened out and fitted into a step-wise multivariate Cox regression to construct the gene signature. HR was utilized to determine the risky genes (HR > $1)$ and protective genes $(\mathrm{HR}<1)$. Subsequently, the risk score for each patient was computed using the following equation:

$$
\text { Risk score }=\sum_{i=1}^{n} E_{i} * \beta_{i}
$$

where " $n$ " is the number of the selected genes, "Ei" stands for the expression level of gene $i$, and " $\beta i$ " represents the coefficient of gene $\mathrm{i}$.

Patients were classified into low- and high-risk groups according to the median risk score (Zhou et al., 2016). Meanwhile, the prognostic performance of the risk score was measured using the time-dependent receiver operating characteristic (ROC) curves by calculating the area under the curve (AUC) using the R package "survivalROC" (Heagerty et al., 2000). The defining point set up by 1-, 2-, 3-, 4- and 5 -year time-dependent ROC curve analysis was employed to assess the predictive value of the risk score for timedependent outcomes (Heagerty et al., 2000). Survival curves in the low- and high-risk groups were plotted by means of the
Kaplan-Meier methods and the differences in the survival time between the two groups were compared using the Log-Rank test and Cox regression analysis (Jones and Crowley, 1989).

\section{Cox regression analysis of the prognostic signature and other clinical parameters}

Influences of various variables including risk score, age, gender, and stage on OS were evaluated by univariate and multivariate Cox proportional hazard regression models.

\section{Results}

\section{Identification of methylation associated DEGs}

To screen out the DEGs between the LUAD and the paired adjacent normal samples, both EdgeR and DEseq2 packages were used. A total of 9384 DEGs were detected by using the EdgeR package according to the threshold of fold change $>1$ and an FDR value $<0.01$, of which 6163 were up-regulated and 3221 were down-regulated. Meanwhile, 8894 DEGs were identified by using the DESeq 2 package, of which 5390 were up-regulated and 3504 were down-regulated. The distribution of the DEGs identified by both EdgeR and DEseq2 were shown using volcano plots (Figure 2A). Unsupervised hierarchical clustering analysis showed that these DEGs could distinguish LUAD samples and the adjacent normal samples (Figure 2B). Similarly, the PCA analysis also suggested that these DEGs could separate samples into LUAD and normal (Figure 2C). Finally, a total of 8495 DEGs were screened out (5303 up- and 3192 down-regulated) by overlapping the DEGs extracted by EdgeR and DEseq2 package.

To identify the methylation associated genes, we analyzed the correlation between the gene expression and the level of DNA methylation. Based on the predefined criteria $(\mathrm{R}<-0.5$ and $\mathrm{P}<0.05)$, a total of 634 methylation associated genes were extracted. Subsequently, the common part between the 634 methylation associated genes and the 8495 DEGs were extracted. Ultimately, 210 methylationassociated DEGs were identified for prognosis analysis, of which 159 was up-regulated (Figure 2D) and 51 was downregulated (Figure 2E).

\section{Identification of risky and protective genes}

To evaluate the prognostic value of the methylationassociated DEGs, a univariate Cox regression analysis was conducted to investigate the correlation between the expression level of these methylation associated DEGs and the OS of the LUAD patients. Consequently, 21 methylation-associated DEGs (12 up- and 9 down-regulated) were found to be significantly associated with the OS. As shown in Figure S1A and S1B, the HRs of these 12 up-regulated DEGs were more than 1 (risky genes), while those of the 9 down-regulated DEGs were less than 1 (protective genes). All these 21 DEGs showed a negative correlation between the DNA methylation status and the gene expression level (Figure S2). The 12 upregulated DEGs with hypomethylation might be risky genes (Figure S2A), whereas the other 9 down-regulated DEGs with hypermethylation might be protective genes (Figure S2B). 

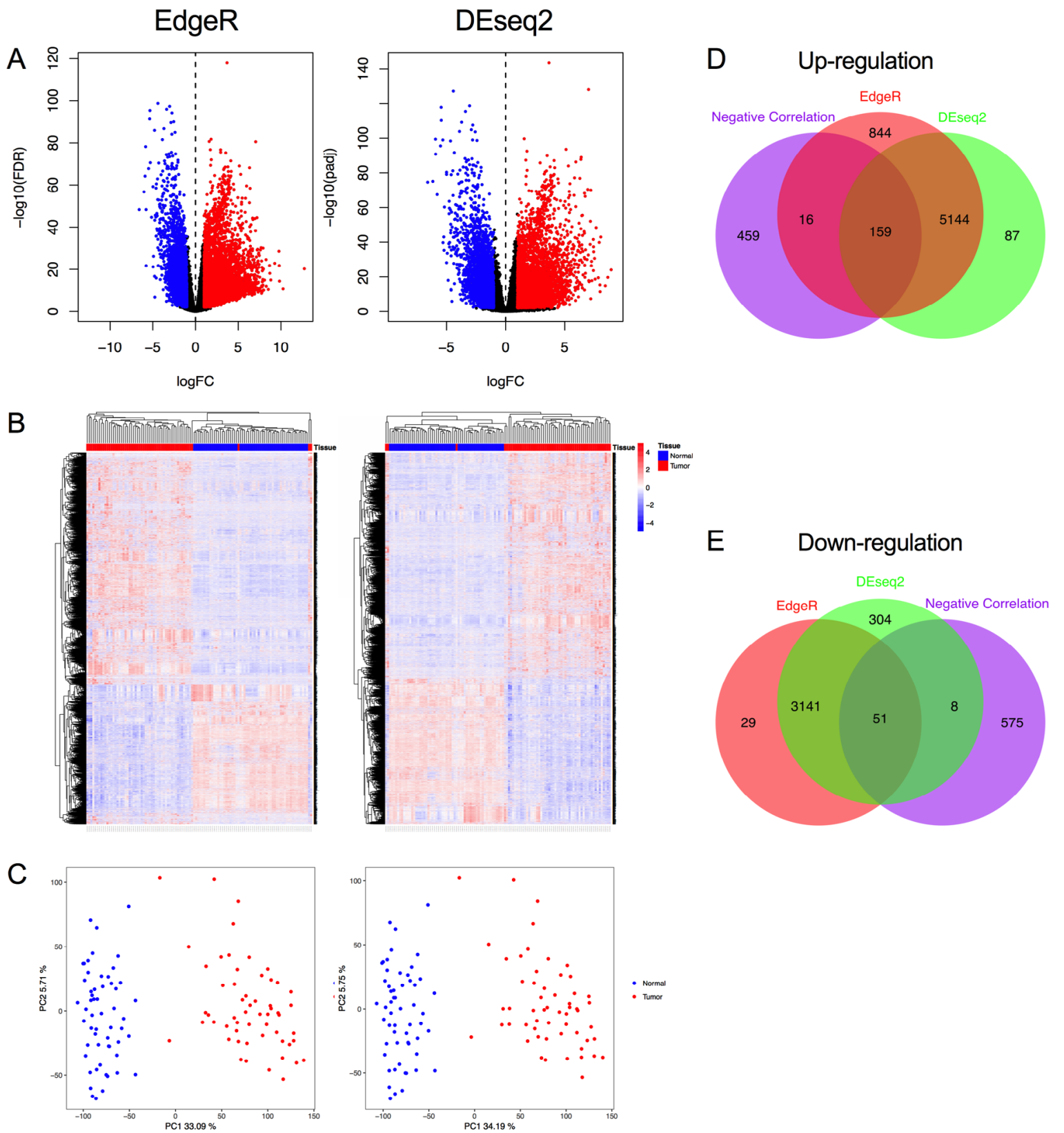

Figure 2 - Identification of methylation-associated differentially expressed genes (DEGs) in lung adenocarcinoma (LUAD). (A) Volcano plots of DEGs analyzed by edgeR and DESeq2. (B) Heatmaps of DEGs identified by edgeR and DESeq2 (C) Principal component analysis (PCA) of the DEGs identified by edgeR and DESeq2. (D) Overlapping of up-regulated DEGs and the genes list negatively correlated with the DNA methylation. (E) Overlapping of down-regulated DEGs and the genes list negatively correlated with the DNA methylation.

\section{Identification of a 6-gene prognostic signature and validation of the risk scoring system based on this gene signature}

Subsequently, a step-wise multivariate Cox model was used to conduct a gene signature. Ultimately, a 6-gene signature (including CCL20, F2, GNPNAT1, NT5E, B3GALT2, and VSIG2) was developed (Table 1). Among these 6 genes, 4 genes (CCL20, F2, GNPNAT1, and NT5E) were unfavorable genes $(\mathrm{HR}>1)$ and the 2 genes (B3GALT2, and VSIG2) were favorable genes $(\mathrm{HR}<1)$.

For each LUAD patient, the risk score was calculated based on the gene expression level and the Cox regression coefficient. According to the threshold of median risk score, 490 patients were divided into a high-risk group and a low-risk group based on the median risk score. The expression pattern of the 6 genes and the survival situation of LUAD patients in the high-risk group and low-risk group were displayed in Figure 3A. From this figure, we found that the mortality rate in the high-risk group was higher, relative to that in the low-risk group.

To investigate the prognostic value of the risk score based on the 6-gene signature in the TCGA LUAD dataset, a univariate analysis was performed. The Kaplan-Meier curves suggested that the OS time of patients in the high-risk group was shorter than that of the low-risk group (Figure 3B, cox $\mathrm{P}=8.42 \mathrm{e}-07$, log-rank $\mathrm{P}=3.33 \mathrm{e}-07$ ), which implicated that the high-risk score was a poor prognostic factor for patients with $\operatorname{LUAD}(\mathrm{HR}=2.61,95 \% \mathrm{CI}=1.78-3.83)$. The prognostic 
Table 1 - Univariate and multivariate analysis of the 6 genes for constructing the prognostic signature.

\begin{tabular}{|c|c|c|c|c|c|c|c|}
\hline \multirow{2}{*}{ Gene symbol } & \multirow{2}{*}{ Ensembl ID } & \multirow{2}{*}{ Gene type } & \multirow{2}{*}{$\begin{array}{l}\text { Chromosomal } \\
\text { position }\end{array}$} & \multicolumn{2}{|c|}{ Univariate analysis } & \multicolumn{2}{|c|}{ Multivariate analysis } \\
\hline & & & & $\mathrm{HR}(95 \% \mathrm{CI})$ & $\mathrm{P}$ & HR $(95 \% \mathrm{CI})$ & $\mathrm{P}$ \\
\hline CCL20 & ENSG00000115009 & Protein coding & $\begin{array}{l}\text { Chr2: } 227805739- \\
\quad 227817564\end{array}$ & $1.11(1.02-1.20)$ & $1.50 \mathrm{E}-02$ & $1.12(1.03-1.22)$ & $7.00 \mathrm{E}-03$ \\
\hline $\mathrm{F} 2$ & ENSG00000180210 & Protein coding & $\begin{array}{l}\text { Chr11: } 46719180- \\
\quad 46739506\end{array}$ & $1.13(1.06-1.21)$ & $2.10 \mathrm{E}-04$ & $1.13(1.05-1.21)$ & $8.20 \mathrm{E}-04$ \\
\hline GNPNAT1 & ENSG00000100522 & Protein coding & $\begin{array}{l}\text { Chr14: } 46719180- \\
\quad 52791668\end{array}$ & $1.65(1.34-2.04)$ & $3.50 \mathrm{E}-06$ & $1.38(1.05-1.80)$ & $1.90 \mathrm{E}-02$ \\
\hline NT5E & ENSG00000135318 & Protein coding & $\begin{array}{l}\text { Chr6: } 46719180- \\
\quad 85495791\end{array}$ & $1.17(1.06-1.31)$ & $3.10 \mathrm{E}-03$ & $1.23(1.11-1.37)$ & $1.60 \mathrm{E}-04$ \\
\hline B3GALT2 & ENSG00000162630 & Protein coding & $\begin{array}{c}\text { Chr1: 46719180- } \\
193186654\end{array}$ & $0.85(0.77-0.95)$ & $4.10 \mathrm{E}-03$ & $0.89(0.79-1.00)$ & $4.40 \mathrm{E}-02$ \\
\hline VSIG2 & ENSG00000019102 & Protein coding & $\begin{array}{c}\text { Chr11: } 46719180- \\
124752238\end{array}$ & $0.88(0.82-0.95)$ & $5.20 \mathrm{E}-04$ & $0.87(0.79-0.97)$ & $1.20 \mathrm{E}-02$ \\
\hline
\end{tabular}

HR: hazard ratio; CI: confidence interval

A

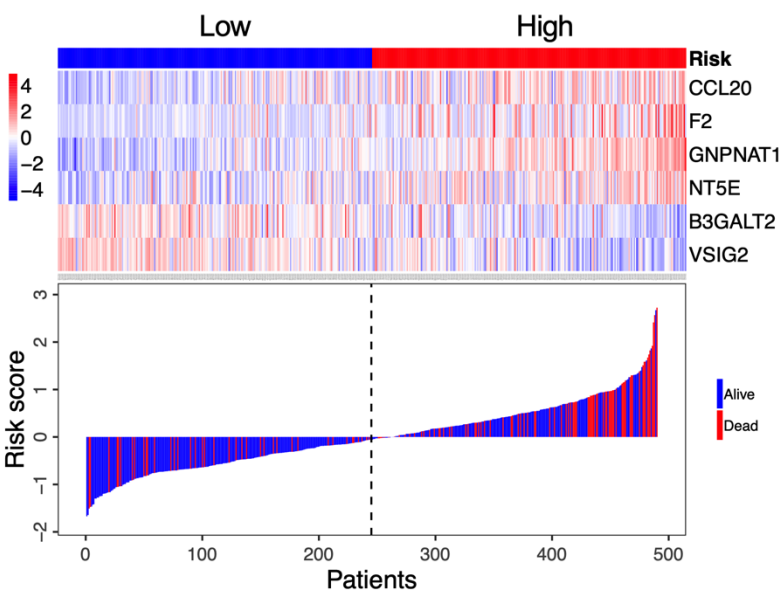

B
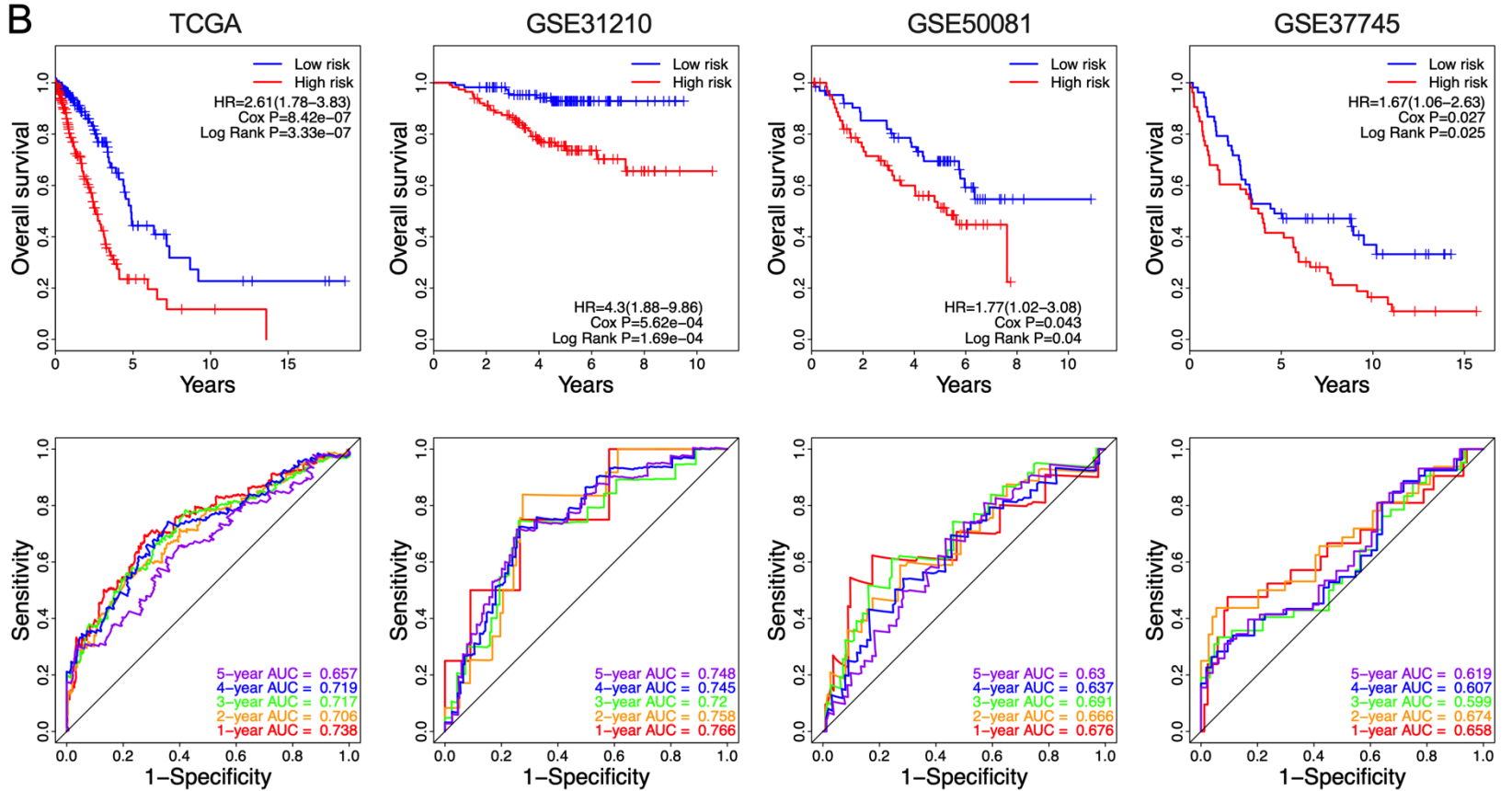

Figure 3 - Construction and validation of the methylation-associated gene signature. (A) The expression patterns of the 6 genes in the high-risk and low-risk group, and the survival status of each LUAD patient in the TCGA data set. The black dotted line is the median risk score, which is utilized to divide patients into the high-risk group and low-risk group. (B) Kaplan-Meier and ROC analysis of the survival prediction performance of the risk score which was based on the methylation-associated gene signature. TCGA was used as a training set, and GSE31210, GSE50081, and GSE37745 were used as validation sets. 
capacity of the risk score was investigated by calculating the AUC value of the ROC curves. The time-dependent ROC curves for 1-, 2-, 3-, 4, and 5-year survival prediction are listed in Figure 3B, with an AUC of 0.738, 0.706, 0.717, 0.719, and 0.657 , respectively, demonstrating that the risk score had a high specificity and sensitivity in predicting of OS.

To investigate the reliability of the risk score for prediction of OS, 3 expression profile datasets including GSE31210, GSE50081 and GSE37745 were used for validation. The results implicated that all patients in the high-risk group had a shorter OS than those in the low-risk group (GSE31210: $\mathrm{HR}=4.3,95 \% \mathrm{CI}=1.88-9.86$, Log-Rank $\mathrm{P}=1.69 \mathrm{e}-04$; GSE50081: $\mathrm{HR}=1.77,95 \% \mathrm{CI}=1.02-3.08$, Log-Rank $\mathrm{P}=$ 0.04; GSE37745: $\mathrm{HR}=1.67,95 \% \mathrm{CI}=1.06-2.63$, Log-Rank $\mathrm{P}=0.025$ ) (Figure 3B). The time-dependent ROC curves showed that the 1-, 2-, 3-, 4-, and 5-year AUC values were $0.766,0.758,0.72,0.745$, and 0.748 in GSE31210, 0.676, $0.666,0.691,0.637$, and 0.63 in GSE50081, 0.658, 0.674, $0.599,0.607$, and 0.619 in GSE37745, respectively (Figure 3B), demonstrating a reliable performance for predicting OS.
The gene expression pattern and the DNA methylation levels of the 6 genes are shown in Figure 4. The expression level of CCL20, F2, GNPNAT1 and NT5E in the tumor samples was significantly higher than that in normal tissues, while the expression level of B3GALT2, and VSIG2 displayed an opposite expression pattern (Figure 4A). Similarly, the expression of CCL20, F2, GNPNAT1 and NT5E was significantly higher in the high-risk group compared to the low-risk groups, but the expression of B3GALT2 and VSIG2 in the high-risk group was lower than that of the low-risk group (Figure 4B). A comparison of the DNA methylation levels of these 6 genes between high- and low-risk groups was performed (Figure 4C). Of note, the methylation level of 3 genes (CCL20, GNPNAT1, and NT5E) was downregulated in the high-risk group compared with the low-risk group (all $\mathrm{P}<0.05$ ). In contrast, the methylation levels of the 2 genes (B3GALT2 and VSIG2) were up-regulated in the high-risk group (both $\mathrm{P}<0.05$ ). However, no difference in DNA methylation level of F2 was observed in the two groups $(\mathrm{P}>0.05)$.
A
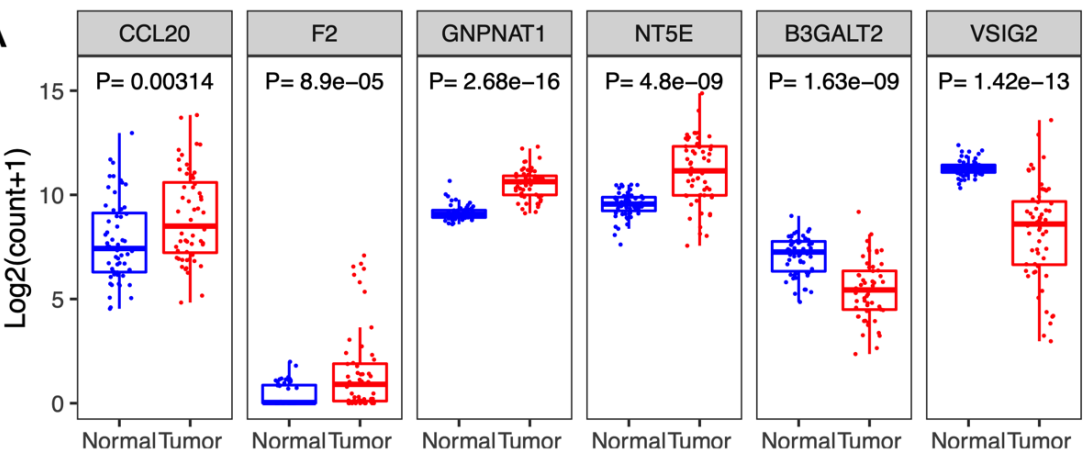

B
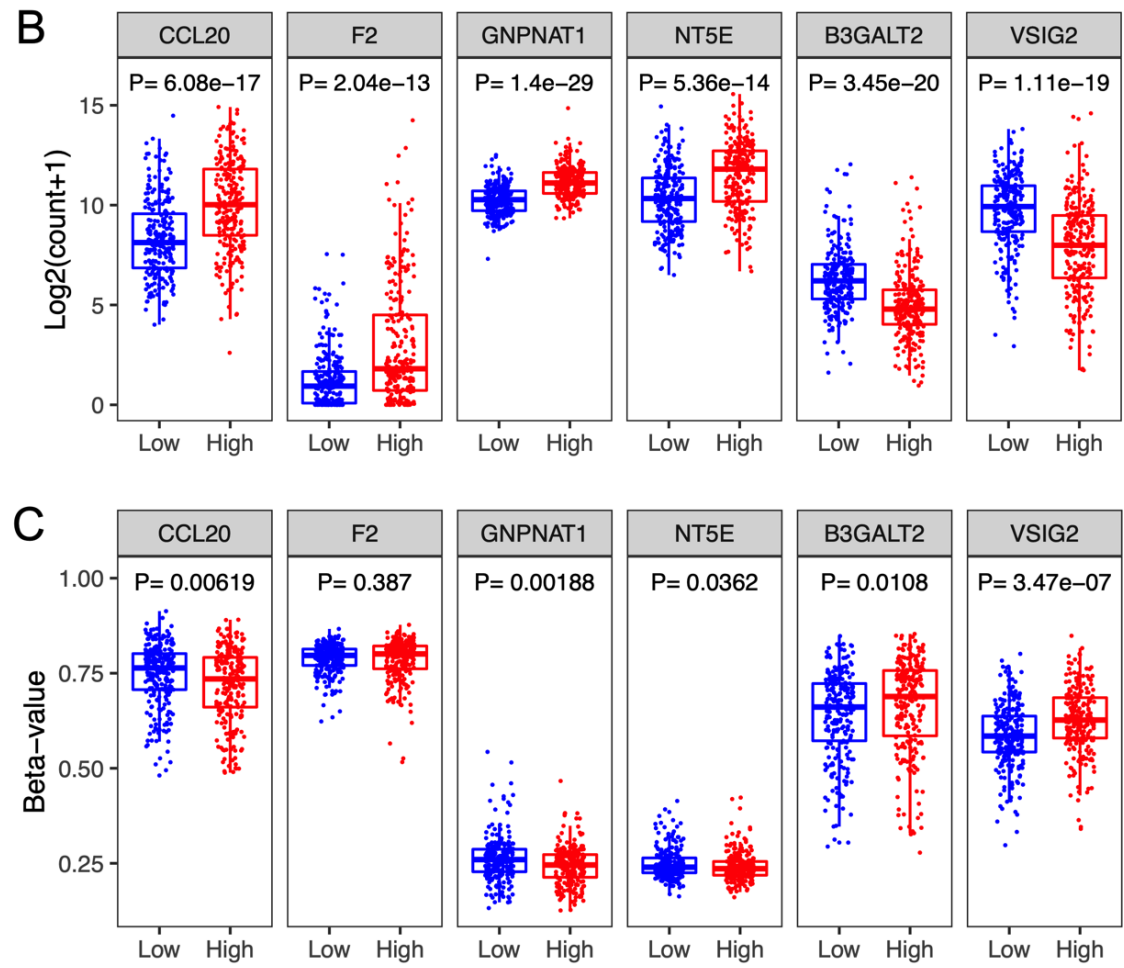

Figure 4 - Expression patterns of methylation-associated gene signature. The expression level of the 6 genes in LUAD and paired adjacent normal tissues (A), and in high-risk and low-risk groups (B). (C) DNA methylation levels (Beta-value) of the 6 genes in high-risk and low-risk groups. 


\section{The risk score is an independent survival predictive} factor

To investigate the prognostic factors for LUAD patients, a univariable Cox analysis was carried out based on the selected variables including age, gender, stage, and risk score. The results showed that stage $\mathrm{II}(\mathrm{HR}=2.68,95 \% \mathrm{CI}=1.68-4.26$, $\mathrm{P}=3.20 \mathrm{E}-05)$, stage III $(\mathrm{HR}=4.39,95 \% \mathrm{CI}=2.77-6.98, \mathrm{P}$ $=3.80 \mathrm{E}-10)$, stage $\mathrm{IV}(\mathrm{HR}=3.22,95 \% \mathrm{CI}=1.64-6.32, \mathrm{P}=$ $6.70 \mathrm{E}-04)$, and high-risk score $(\mathrm{HR}=2.61,95 \% \mathrm{CI}=1.78$ $3.83, \mathrm{P}=8.40 \mathrm{E}-07$ ) were significantly correlated with poor OS of LUAD patients (Table 2).

To measure whether the risk score was independent of other clinical features, multivariable Cox analysis was implemented. The results showed that age $(\mathrm{HR}=1.62,95 \%$ $\mathrm{CI}=1.11-2.37, \mathrm{P}=1.20 \mathrm{E}-02)$, stage $\mathrm{II}(\mathrm{HR}=2.60,95 \% \mathrm{CI}=$ $1.62-4.18, \mathrm{P}=7.70 \mathrm{E}-05)$, stage III $(\mathrm{HR}=3.37,95 \% \mathrm{CI}=$ 2.08-5.43, $\mathrm{P}=7.00 \mathrm{E}-07)$, stage $\mathrm{IV}(\mathrm{HR}=3.69,95 \% \mathrm{CI}=$ $1.86-7.32, \mathrm{P}=2.00 \mathrm{E}-04)$, and high risk score $(\mathrm{HR}=2.30,95 \%$ $\mathrm{CI}=1.53-3.46, \mathrm{P}=6.30 \mathrm{E}-07)$ were independent prognostic factors for LUAD patients (Table 2).

A stratification analysis was further performed based on clinical parameters. Patients in each subgroup including age $(<=65$, and $>65)$, gender (male and female), and stage (II, and III-IV) were separated into the low-risk group and high-risk group according to the median risk score. For all stratified clinical variables, patients in the high-risk group had a shorter survival time, relative to those of the low-risk group (Figure 5, Log-Rank $\mathrm{P}<0.05$, Cox $\mathrm{P}<0.05$ ). Taken together, these findings suggested that the risk score based on the 6-gene signature was an independent survival predictive factor.

\section{Discussion}

DNA methylation can regulate the gene expression and usually induces tumor suppressor gene silencing and oncogene activation through hyper/hypomethylation (Belinsky, 2004; Vaissiã Re et al., 2008). In this study, we demonstrated for the first time that integrated analysis of gene expression profiles and DNA methylation data could establish methylation-associated gene signature that can be used to predict the survival of LUAD patients. The risk score based on the methylation-associated gene signature exhibited good predictive performance in both TCGA and GEO datasets.

Our methylation-associated gene signature showed comparable sensitivity and specificity to the previous study for survival prediction (Figure S3). In a previous study (Lau et al., 2007), a 3-gene signature classified the patients into two groups and showed different survival times, however, the AUC values of the gene signature were not assessed. Although an 8-gene signature (He and Zuo, 2019) performed better in predicting survival in NSCLC patients, it did not perform as robustly as the gene signature in our study for predicting LUAD. A similar predictive performance was observed between a 7-gene signature (Krzystanek et al., 2016) and our methylation-associated gene signature, nevertheless, the AUC in their study was not calculated. In a 4-gene signature (Cui et al., 2019), the predictive performance based on the TCGA dataset was inferior to our signature, and the AUC values were not validated. Although all signatures were capable of predicting OS, our methylation-associated gene signature was much more robust.

In this methylation-associated gene signature, the expression level of CCL20, F2, GNPNAT1, and NT5E was significantly up-regulated in the LUAD tissue and in the high-risk group. Significantly, the HRs of these 4 up-regulated genes were more than 1 , and those methylation levels were hypomethylated, which indicated that these hypomethylatedup-regulation genes are risky genes. Chemokines are responsible for the establishment of the tumor microenvironment, and the infiltration and migration of inflammatory cells and cancer cells (King, 2015). CCL20, a member of CC chemokines, has been observed to mediate the migration of inflammatory cells, thereby involving in metastasis of cancer, including colorectal, pancreatic, or lung cancer (Beider et al., 2009; Brand et al., 2010; Wang et al., 2016). Moreover, Wang et al. (2015) have demonstrated that CCL20 is up-regulated in lung cancer, and increased CCL20 is related to poor prognosis. The full name for F2 is coagulation factor II which has been reported to be a prerequisite for lung-cancer-cell-induced platelet aggregation (Heinmöller et al., 1996). Significantly, in some instances, platelet aggregation directly links with the metastatic potential (Tang and Honn, 1995). GNPNAT1 was only reported in prostate cancer, which was suggested to be over-expressed in prostate cancer tissue (Ren et al., 2016) and to be connected with the development of castrationresistant prostate cancer (Kaushik et al., 2016). Growing evidence shows that NT5E is a key regulatory molecule in the development of cancer and is highly expressed in a number of cancers, including NSCLC (Zhu et al., 2017), and silence of NT5E suppresses the cell growth and migration of NSCLC cells (Zhu et al., 2017). Significantly, high NT5E expression was an independent predictor of poor prognosis for OS and recurrence-free survival in NSCLC (Inoue et al., 2017). Another two genes identified

Table 2 - Univariate and multivariate Cox regression analysis of the 6-gene signature and overall survival of LUAD patients.

\begin{tabular}{|c|c|c|c|c|c|c|}
\hline \multirow{2}{*}{ Variables } & & \multirow{2}{*}{$\begin{array}{l}\text { Patients } \\
\text { (N) }\end{array}$} & \multicolumn{2}{|c|}{ Univariate analysis } & \multicolumn{2}{|c|}{ Multivariate analysis } \\
\hline & & & HR $(95 \% \mathrm{CI})$ & $\mathrm{P}$ & HR $(95 \%$ CI $)$ & $\mathrm{P}$ \\
\hline Age & $<=65 />65$ & $237 / 253$ & $1.35(0.94-1.95)$ & $1.00 \mathrm{E}-01$ & $1.62(1.11-2.37)$ & $1.20 \mathrm{E}-02$ \\
\hline Gender & Male/Female & $265 / 225$ & $0.93(0.65-1.33)$ & $6.90 \mathrm{E}-01$ & $0.79(0.55-1.15)$ & $2.20 \mathrm{E}-01$ \\
\hline Stage & $\mathrm{I} / \mathrm{II}$ & $262 / 115$ & $2.68(1.68-4.26)$ & $3.20 \mathrm{E}-05$ & $2.60(1.62-4.18)$ & $7.70 \mathrm{E}-05$ \\
\hline Stage & I/III & $262 / 80$ & $4.39(2.77-6.98)$ & $3.80 \mathrm{E}-10$ & $3.37(2.08-5.43)$ & $7.00 \mathrm{E}-07$ \\
\hline Stage & $\mathrm{I} / \mathrm{IV}$ & $262 / 26$ & $3.22(1.64-6.32)$ & $6.70 \mathrm{E}-04$ & $3.69(1.86-7.32)$ & $2.00 \mathrm{E}-04$ \\
\hline Risk score & Low/High & $245 / 245$ & $2.61(1.78-3.83)$ & $8.40 \mathrm{E}-07$ & $2.30(1.53-3.46)$ & $6.30 \mathrm{E}-05$ \\
\hline
\end{tabular}

HR: hazard ratio; CI: confidence interval 

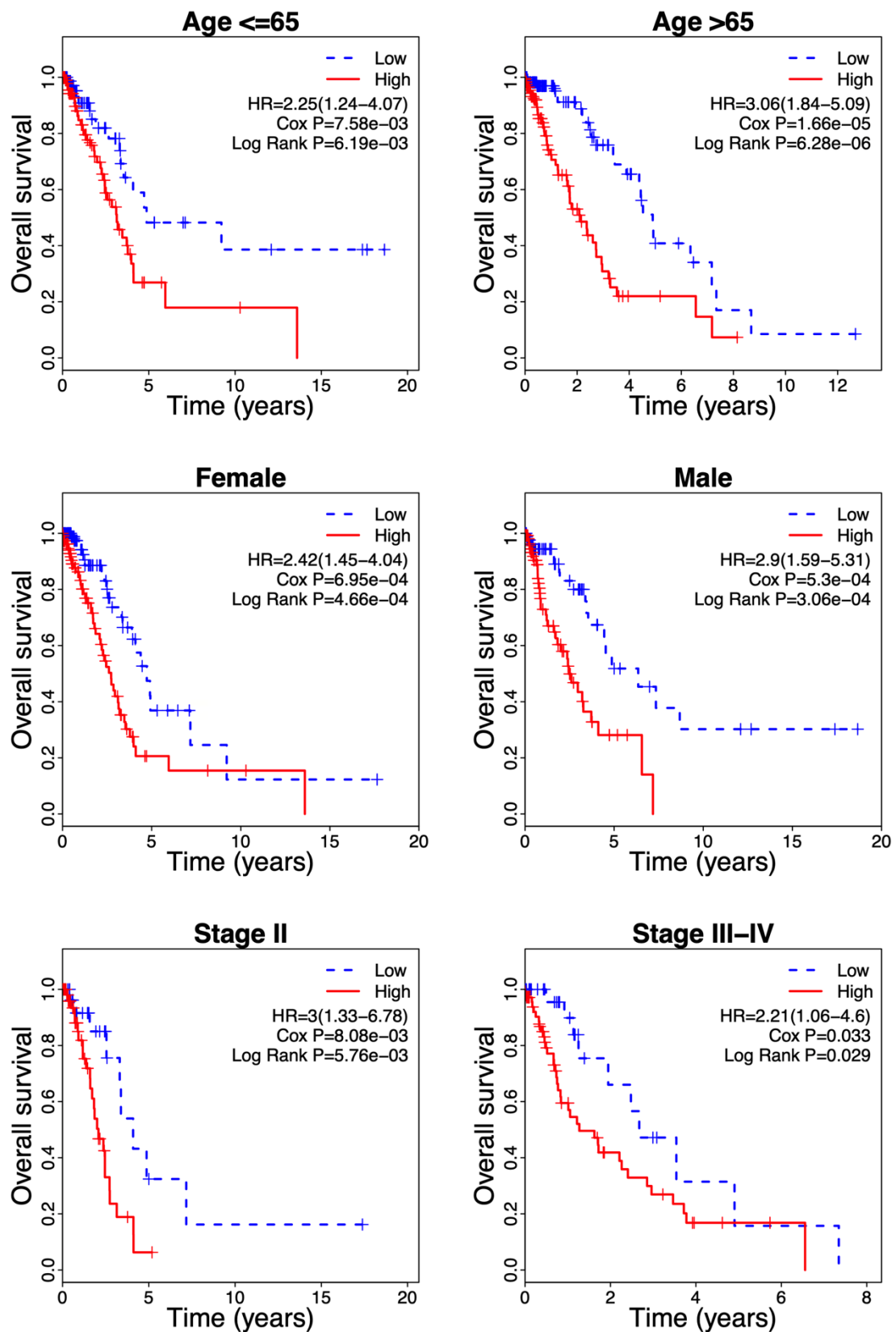

Figure 5 - Stratification analysis of the survival prediction performance of the risk score in different clinical parameters of LUAD patients. HR, hazard ratio.

in our prognostic signature are B3GALT2 and VSIG2 which were down-regulated in the LUAD samples and in the high-risk group in our study. Moreover, those HR values were less than 1 , and these two genes were hypermethylated, suggesting genes B3GALT2 and VSIG2 with hypermethylated-down-regulation were protective genes. In a former study, 1 down-regulated gene B3GALT2 was identified among 139 LUAD-specific hypermethylated genes (Yin et al., 2014), which is in line with our results. B3GALT2 is applied to form a prognostic biomarker of carcinoma-associated fibroblasts in NSCLC (Navab et al., 2011). Additionally, carcinoma-associated fibroblasts play a crucial role in maintaining an optimal cancer microenvironment for cell proliferation and survival (Cirri and Chiarugi, 2012; Marsh et al., 2013). VSIG2 has been found to be differentially expressed in endometrial cancer (Shi et al., 2018), and to be significantly associated with bladder cancer risk (Moore et al., 2010). Since the 6-gene signature is established based on the hypomethylation-related risky genes and hypermethylation-related protective genes, it can provide new ideas for methylation-based treatment for LUAD. For example, the methylation strategy of a hypomethylated risky gene, or the demethylation of a hypermethylated protective gene in the signature.

Nevertheless, several disadvantages should be acknowledged in the current study. Firstly, this study is a retrospective study based on previously published datasets, hence, prospective studies should be carried out in the future to remedy the limitations of the retrospective study. Secondly, the functions of these methylation-associated DEGs should be verified based on experimental investigations. Thirdly, clinical studies are needed to further verify the accuracy and application potential of this novel prognostic signature for LUAD patients. 


\section{Conclusion}

The risk score based on the methylation-associated gene signature is an independent survival predictive factor for LUAD patients. The potential clinically applicable methylation-associated gene signature may contribute to improving the prognosis of LUAD patients and the represent hypermethylated or hypomethylated genes might be potential targets for LUAD therapy.

\section{Conflict of Interest}

The authors declare that they have no financial and non-financial interests.

\section{Author Contributions}

MJ and QZG conceived and the study, CLC and CL analyzed the data, MJ and SHF wrote the manuscript. All authors read and approved the final version of the manuscript.

\section{References}

Anders S and Huber W (2010) Differential expression analysis for sequence count data. Genome Biol 11:R106

Beider K, Abraham M, Begin M, Wald H, Weiss ID, Wald O, Pikarsky E, Abramovitch R, Zeira E and Galun E (2009) Interaction between CXCR4 and CCL20 pathways regulates tumor growth. PloS One 4:e5125

Belinsky SA(2004) Gene-promoter hypermethylation as a biomarker in lung cancer. Nat Rev Cancer 4:707-717

Benjamini Y, Dan D, Elmer G, Kafkafi N and Golani I (2001) Controlling the false discovery rate in behavior genetics research. Behav Brain Res 125:279-284

Bjaans MM, Fleischer T, Halvorsen AR, Daunay A, Busato F, Solberg S, Jrgensen L, Kure E, Edvardsen H, Brresen-Dale AL et al. (2016) Genome-wide DNA methylation analyses in lung adenocarcinomas: Association with EGFR, KRAS and TP53 mutation status, gene expression and prognosis. Mol Oncol 10:330-343

Botling J, Edlund K, Lohr M, Hellwig B, Holmberg L, Lambe M, Berglund A, Ekman S, Bergqvist M and Ponten F (2013). Biomarker discovery in non-small cell lung cancer: Integrating gene expression profiling, meta-analysis, and tissue microarray validation. Clin Cancer Res 19:194-204

Brand S, Olszak T, Beigel F, Diebold J, Otte JM, Eichhorst ST, Göke B and Dambacher J (2010) Cell differentiation dependent expressed CCR6 mediates ERK-1/2, SAPK/JNK, and Akt signaling resulting in proliferation and migration of colorectal cancer cells. J Cell Biochem 97:709-723

Brock MV, Hooker CM, Ota-Machida E, Han Y, Guo M, Ames S, Glockner S, Piantadosi S, Gabrielson E, Pridham G et al. (2008) DNA methylation markers and early recurrence in stage I lung cancer. N Engl J Med 358:1118-1128

Chen H and Boutros PC (2011) VennDiagram: a package for the generation of highly-customizable Venn and Euler diagrams in R. BMC bioinformatics 12:35

Cirri P and Chiarugi P (2012) Cancer-associated-fibroblasts and tumour cells: A diabolic liaison driving cancer progression. Cancer Metast Rev 31:195-208

Cui Y, Fang W, Li C, Tang K, Zhang J, Lei Y, He W, Peng S, Kuang M, Zhang H et al. (2019) Development and validation of a novel signature to predict overall survival in "Driver Gene-negative" Lung Adenocarcinoma (LUAD): Results of a multicenter study. Clin Cancer Res 25:1546-1556
Der SD, Sykes J, Pintilie M, Zhu CQ, Strumpf D, Liu N, Jurisica I, Shepherd FA and Tsao MS (2014) Validation of a histologyindependent prognostic gene signature for early-stage, nonsmall-cell lung cancer including stage IA patients. J Thorac Oncol 9:59-64

Gharibvand L, Beeson WL, Shavlik D, Knutsen R, Ghamsary M, Soret S and Knutsen SF (2017) The association between ambient fine particulate matter and incident adenocarcinoma subtype of lung cancer. Environ Health 16:71

Hao X, Luo H, Krawczyk M, Wei W and Zhang K (2017) DNA methylation markers for diagnosis and prognosis of common cancers. P Natl Acad Sci U S A 114:7414-7419

He R and Zuo S (2019) A Robust 8-Gene Prognostic Signature for Early-Stage Non-small Cell Lung Cancer. Front Oncol 9:693

Heagerty PJ, Lumley T and Pepe MS (2000) Time-dependent ROC curves for censored survival data and a diagnostic marker. Biometrics 56:337-344

Heinmöller E, Weinel RJ, Heidtmann HH, Salge U, Seitz R, Schmitz I, Müller KM and Zirngibl H (1996) Studies on tumor-cellinduced platelet aggregation in human lung cancer cell lines. J Cancer Res Clin 122:735-744

Inoue Y, Yoshimura K, Kurabe N, Kahyo T, Kawase A, Tanahashi M, Ogawa H, Inui N, Funai K and Shinmura K (2017) Prognostic impact of CD73 and A2A adenosine receptor expression in non-small-cell lung cancer. Oncotarget 8:8738-8751

Jones MP and Crowley J (1989) A general class of nonparametric tests for survival analysis. Biometrics 45:157-170

Kaushik AK, Shojaie A, Panzitt K, Sonavane R, Venghatakrishnan H, Manikkam M, Zaslavsky A, Putluri V, Vasu VT and Zhang $Y$ (2016) Inhibition of the hexosamine biosynthetic pathway promotes castration-resistant prostate cancer. Nat Commun 7:11612

King PT (2015) Inflammation in chronic obstructive pulmonary disease and its role in cardiovascular disease and lung cancer. Clin Transl Med 4:26

Krzystanek M, Moldvay J, Szüts D, Szallasi Z and Eklund AC (2016) A robust prognostic gene expression signature for early stage lung adenocarcinoma. Biomark Res 4:4

Lau SK, Boutros PC, Pintilie M, Blackhall FH, Zhu CQ, Strumpf D, Johnston MR, Darling G, Keshavjee S and Waddell TK (2007) Three-gene prognostic classifier for early-stage non small-cell lung cancer. J Clin Oncol 25:5562-5569

Li YF, Wang Y, Li JL, Hao XZ and Wang HY (2016). Trend analysis and clinicopathological characteristics of 198 young patients with advanced lung adenocarcinoma. Zhonghua Zhong Liu Za Zhi 38:750

Marsh T, Pietras K and McAllister SS (2013) Fibroblasts as architects of cancer pathogenesis. BBA-Mol Basis Dis 1832:1070-1078

Moore LE, Malats N, Rothman N, Real FX, Kogevinas M, Karami S, García-Closas R, Silverman D, Chanock S and Welch R (2010) Polymorphisms in one-carbon metabolism and transsulfuration pathway genes and susceptibility to bladder cancer. Int J Cancer 120:2452-2458

Navab R, Strumpf D, Bandarchi B, Zhu CQ, Pintilie M, Ramnarine VR, Ibrahimov E, Radulovich ND, Leung L, Barczyk M et al. (2011) Prognostic gene-expression signature of carcinomaassociated fibroblasts in non-small cell lung cancer. P Natl Acad Sci U S A 108:7160-7165

Okayama H, Kohno T, Ishii Y, Shimada Y, Shiraishi K, Iwakawa R, Furuta K, Tsuta K, Shibata T and Yamamoto S (2012) Identification of genes upregulated in ALK-positive and EGFR/KRAS/ALK-negative lung adenocarcinomas. Cancer Res 72:100-111 
$\mathrm{Pu}$ W, Geng X, Chen S, Tan L and Wang J (2016) Aberrant methylation of $\mathrm{CDH} 13$ can be a diagnostic biomarker for lung adenocarcinoma. J Cancer 7:2280-2289

Ren S, Shao Y, Zhao X, Hong CS, Wang F, Lu X, Li J, Ye G, Yan M and Zhuang Z (2016) Integration of metabolomics and transcriptomics reveals major metabolic pathways and potential biomarker involved in prostate cancer. Mol Cell Proteomics 15:154

Robinson MD, McCarthy DJ and Smyth GK (2009) Robinson MD, McCarthy DJ, Smyth GK.. EdgeR: a Bioconductor package for differential expression analysis of digital gene expression data. Bioinformatics 26: 139-140. Bioinformatics 26:139-140

Selamat SA, Chung BS, Girard L, Zhang W, Zhang Y, Campan M, Siegmund KD, Koss MN, Hagen JA, Lam WL et al. (2012) Genome-scale analysis of DNA methylation in lung adenocarcinoma and integration with mRNA expression. Genome Res 22:1197-1211

Sheikhnejad R, Zohri M, Shadmehr MB, Rahmani-Khalili M, Doozande D, Farsad Z and Sheikhzade K (2013) Detection of aberrant methylation of 10 genes in genomic DNA of lung tumors. Ann Oncol 24:2705-2706

Shi Z, Li C, Laura T, Li J, Yang L, William K, Chandrashekar DS and Sharon SM (2018). RNA-seq reveals the overexpression of IGSF9 in endometrial cancer. J Oncol 2018:2439527

Siegel RL, Miller KD and Jemal A (2015) Cancer statistics, 2015. CA Cancer J Clin 65:5-29

Swm A, Hjsa B, Hym A, Ejc A, Njy A and Shla B (2021) Mutation and expression alterations of histone methylation-related NSD2, KDM2B and SETMAR genes in colon cancers. Pathol Res Pract 219:153354.

Tang DG and Honn KV (1995) Adhesion molecules and tumor metastasis: An update. Invasion Metastasis 14:109-122

Vaissiã Re T, Sawan C and Herceg Z (2008) Epigenetic interplay between histone modifications and DNA methylation in gene silencing. Mutat Res 659:40-48

Wang B, Shi L, Sun X, Wang L, Wang X and Chen C (2016) Production of CCL20 from lung cancer cells induces the cell migration and proliferation throughPI3K pathway. J Cell Mol Med 20:920-929
Wang GZ, Cheng X, Li XC, Liu YQ, Wang XQ, Shi X, Wang ZY, Guo YQ, Wen ZS, Huang YC et al. (2015) Tobacco smoke induces production of chemokine CCL20 to promote lung cancer. Cancer Lett 363:60-70

Xu Rh, Wei W, Krawczyk M, Wang W, Luo H, Flagg K, Yi S, Shi W, Quan Q and Li K (2017) Circulating tumour DNA methylation markers for diagnosis and prognosis of hepatocellular carcinoma. Nat Mater 16:1155

Yamashita K, Hosoda K, Nishizawa N, Katoh H and Watanabe M (2018) Epigenetic biomarkers of promoter DNA methylation in the new era of cancer treatment. Cancer Sci 109:3695-3706

Yin LG, Zou ZQ, Zhao HY, Zhang CL, Shen JG, Qi L, Qi M and Xue ZQ (2014) Analysis of tissue-specific differentially methylated genes with differential gene expression in non-small cell lung cancers. Mol Biol 48:694-700

Zhou X, Huang Z, Xu L, Zhu M, Zhang L, Zhang H, Wang X, Li $\mathrm{H}, \mathrm{Zhu} \mathrm{W}$ and Shu Y (2016) A panel of 13-miRNA signature as a potential biomarker for predicting survival in pancreatic cancer. Oncotarget 7:69616-69624

Zhu J, Zeng Y, Li W, Qin H, Lei Z, Shen D, Gu D, Huang JA and Liu Z (2017) CD73/NT5E is a target of miR-30a-5p and plays an important role in the pathogenesis of non-small cell lung cancer. Mol Cancer 16:34

\section{Supplementary material}

The following online material is available for this article:

Figure S1 - Kaplan-Meier survival curves of DEGs.

Figure S2 - Correlation between gene expression and methylation was determined using Pearson's correlation coefficient (R).

Figure S3 - Kaplan-Meier survival curves and ROC curves of previously published gene signatures.

Associate Editor: Daisy Maria Fávero Salvadori

License information: This is an open-access article distributed under the terms of the Creative Commons Attribution License (type CC-BY), which permits unrestricted use, distribution and reproduction in any medium, provided the original article is properly cited. 